\title{
Atorvastatin in Pulmonary Arterial Hypertension (APATH) study
}

\author{
Wei-Jie Zeng*», Chang-Ming Xiong*,\$, Lan Zhao ${ }^{\star, \S, ~ G u a n g-L i a n g ~ S h a n `, ~}$ \\ Zhi-Hong Liu*, Fang Xue ", Qing Gu*, Xin-Hai Ni*, Zhi-Hui Zhao*, Xian-Sheng Cheng*, \\ Martin R. Wilkins ${ }^{\#}$ and Jian-Guo He* on behalf of the Atorvastatin in Pulmonary \\ Arterial Hypertension (APATH) Study Group ${ }^{+}$
}

ABSTRACT: Statins have been shown to both prevent and attenuate pulmonary hypertension in animal models. This study investigates the potential therapeutic benefits of atorvastatin as an affordable treatment for pulmonary hypertension patients.

220 patients with pulmonary arterial hypertension (PAH) or chronic thromboembolic pulmonary hypertension (CTEPH) were randomised, double-blind, to receive atrovastatin $10 \mathrm{mg}$ daily or matching placebo in addition to supportive care.

At 6 months, 6-min walk distance decreased by $16.6 \mathrm{~m}$ in the atorvastatin group and $14.1 \mathrm{~m}$ in the placebo group. The mean placebo-corrected treatment effect was $-2.5 \mathrm{~m} \mathrm{(95 \%} \mathrm{Cl:}-38-33$; $\mathbf{p}=\mathbf{0 . 9 6 )}$, based on intention to treat. A small nonsignificant increase in pulmonary vascular resistance and fall in cardiac output was seen in both treatment groups. There was no significant difference in the proportion of patients who improved, remained stable or showed a deterioration in World Health Organization functional class between atorvastatin and placebo treatments. Nine patients died in the atorvastatin group and 11 in the placebo group. Serum cholesterol levels fell significantly on atorvastatin treatment. Discontinuation rates were $23.2 \%$ and $26.9 \%$ on atorvastatin and placebo, respectively.

Atorvastatin $10 \mathrm{mg}$ daily has no beneficial effect on the natural history of PAH or CTEPH over 6 months.

KEYWORDS: Exercise, haemodynamics, hypertension, pulmonary, statin

$\mathbf{T}$ he treatment of pulmonary arterial hypertension $(\mathrm{PAH})$ is a continuing challenge, likewise, the treatment of pulmonary hypertension secondary to inoperable chronic thromboembolic disease [1]. Despite advances in treatment, mortality remains unacceptably high [2-4]. In addition, the current therapeutic options, prostenoids, endothelin receptor antagonists and phosphodiesterase-type 5 inhibitors, are expensive and not widely available in developing countries. In China, only iloprost and bosentan are licensed for PAH. Sildenafil is only licensed for erectile dysfunction. Many patients in China have no access to approved PAH treatments. There is a need for more effective, affordable drugs treatments.

The licensed treatments for pulmonary hypertension are drugs designed primarily to reduce pulmonary vascular tone [5]. It is widely appreciated that the major pathology contributing to the chronic increase in pulmonary vascular resistance is remodelling of pulmonary resistance vessels. This has focused thoughts on strategies that target directly the structural changes and the molecular mechanisms that underlie them.

It is in this context that statins have attracted interest. This drug class inhibits 3-Hydroxy-3methylglutaryl (HMG) Co-A reductase, a key enzyme in cholesterol synthesis but essential for the synthesis of isoprenoids (geranylgeranylpyrophosphate and farnesylpyrophosphate), required for the post-translational isoprenylation of Rho and Ras family GTPases [6]. This confers on statins pleotrophic properties, that include antiproliferative, anti-inflammatory, anti-thrombotic and anti-oxidant effects. There is now a substantial body of data from animal models that show both attenuation in the development of and regression of established experimental pulmonary hypertension with statin treatment [7-18]. There is

\section{AFFILIATIONS}

*Pulmonary Vascular Disease State Key Laboratory of Cardiovascular Diseases, Fuwai Hospital, National Center for Cardiovascular Diseases, Chinese Academy of Medical Sciences \& Peking Union Medical College and

"Institute of Basic Medical Sciences, Chinese Academy of Medical Sciences, Beijing, China. \#Experimental Medicine, Imperial College London, Hammersmith Hospital, London, UK.

${ }^{+}$APATH Study Group participants are listed in the Acknowledgements.

${ }^{\S}$ Both authors contributed equally to this manuscript.

CORRESPONDENCE

J-G. He

Pulmonary Vascular Diseases State

Key Laboratory of Cardiovascular

Diseases

Fuwai Hospita

National Center for Cardiovascular Diseases

Chinese Academy of Medical Sciences and Peking Union Medical College Beijing 100037 China E-mail: hejianguofw@gmail.com

Received:

Aug 282011

Accepted after revision:

Nov 122011

First published online: Feb 232012 
evidence that this is achieved through increased apoptosis as well as reduced proliferation of smooth muscle cells in obstructive vascular lesions.

Data from human studies are few. A series of case reports of patients treated with simvastatin suggested this drug was well-tolerated and may have beneficial effects in licenced doses [19]. The results of two small randomised clinical studies of simvastatin in human PAH have been disappointing with no evidence of an improvement in 6-min walk distance [20, 21]. A study with rosuvastatin has reported a sustained reduction in P-selectin levels and a small $(15 \mathrm{~m})$ improvement in 6-min walk distance at 6 months in a small mixed cohort of patients with pulmonary hypertension of different aetiologies [22].

There are no data on the effect of statins on pulmonary haemodynamics in patients. To understand further the potential therapeutic benefits of statins as a treatment for pulmonary hypertension patients, we conducted a randomised, doubleblind, placebo-controlled study of the effects of atorvastatin $10 \mathrm{mg}$ daily for 6 months on exercise capacity and pulmonary haemodynamics.

\section{METHODS}

\section{Selection of patients}

This study included pulmonary hypertension patients 18-65 years of age with a diagnosis of group $1 \mathrm{PAH}$ (specifically, idiopathic and hereditable $\mathrm{PAH}, \mathrm{PAH}$ associated with connective tissue disease and $\mathrm{PAH}$ associated with congenital heart disease) or inoperable chronic thromboembolic pulmonary hypertension. Patients with $\mathrm{PAH}$ associated with congenital heart disease were enrolled if they had persistent PAH five years after surgical or interventional repair, or if they were not eligible for surgical or interventional treatment. $\mathrm{PAH}$ was defined as mean pulmonary artery pressure more than $25 \mathrm{mmHg}$, pulmonary capillary wedge pressure $<15 \mathrm{mmHg}$ and pulmonary vascular resistance $>3$ Wood units.

The exclusion criteria were as follows. 1) Groups 2, 3 and 5 pulmonary hypertension; 2) a forced expiratory volume in $1 \mathrm{~s} /$ forced vital capacity $<50 \%$ or a total lung capacity of $<60 \%$ predicted value; 3) a 6-min walk distance of $<100 \mathrm{~m}$ or $>460 \mathrm{~m}$; 4) a positive acute vasodilator response; 5) current treatment with calcium-channel blockers or PAH-targeted therapy (endothelin receptor antagonist, phosphodiesterase-5 inhibitor or prostacyclin); 6) inability to perform 6-min walk test; 7) serum transaminase level three-times above the upper limit of normal; 8) creatine kinase level five-times above the upper limit of normal; 9) previously diagnosed heart disease, such as serious cardiac arrhythmias, unstable angina pectoris, myocardial infarction; 10) history of transient ischaemia attack or stroke within 3 months; 11) bleeding disorder; 12) positive pregnancy test or lactatin; or 13) history or suspicion of inability to cooperate.

This study was conducted according to the Declaration of Helsinki and in adherence to good clinical practice guidelines and was approved by the Institutional Review Boards of $\mathrm{Fu}$ Wai Hospital. All patients participated in the study on a voluntary basis after they had been fully informed of the therapy for PAH available to them. Written informed consent was obtained from all patients.

\section{Study design}

This was a 24-week, randomised, double-blind and placebocontrolled trial, conducted in 26 centres in China between May 2007 and March 2010. Using a block randomisation technique with block sizes of four, 220 patients were assigned to receive $10 \mathrm{mg}$ of atorvastatin or matching placebo once daily for 24 weeks (JiaLin Pharmaceutical Co., Beijing, China). The randomisation was not stratified for any factors. Randomisation was performed using a randomisation assignment program by SAS 9.1 (SAS Institute Inc., Cary, NC, USA). The dose was adjusted to $5 \mathrm{mg}$ daily if serum transaminase levels increased by less than three-times the upper limit of normal or creatine kinase levels increased to less than five-times the upper limit of normal. If serum transaminase and creatine kinase levels remained normal and low-density lipoprotein level greater than $3.4 \mathrm{mmol} \cdot \mathrm{L}^{-1}$ after 4 weeks of therapy, the dose of atorvastatin was increased to $20 \mathrm{mg}$ once daily. Blinding continued until all analyses were completed.

\section{Outcome measures}

The primary end-point of the study was the placebo-corrected change from baseline to week 24 in 6-min walk distance. The 6-min walk testing was performed according to the American Thoracic Society guidelines [23]. Secondary end-points included: time from randomisation to clinical worsening (defined as death, the first occurrence of hospitalisation for PAH or initiation of PAH targeted therapy decided by an independent panel of physicians) and change from baseline to week 24 in World Health Organization (WHO) functional class (an adaptation of the New York Heart Association classification [24]), Borg dyspnoea score (with 0 representing no dyspnoea and 10 maximal dyspnoea [23]), and haemodynamic parameters (mean pulmonary artery pressure, right atrial pressure, cardiac output and pulmonary vascular resistance) derived from right heart catheterisation. Haemodynamic parameters were measured as described previously [25]. Cardiac output was determined using the thermodilution technique or calculated according to the Fick method. Pulmonary vascular resistance was calculated as: (mean pulmonary artery pressure - pulmonary capillary wedge pressure)/pulmonary blood flow. 6-min walk test, evaluation of the Borg dyspnoea score and right heart catheterisation were performed at baseline and at week 24. Physical examinations, assessment of WHO functional class and laboratory tests including a complete blood count and routine chemistry tests (creatinine, transaminases, creatine kinase and lipid profile) were performed at baseline and regular intervals. Investigators recorded adverse events throughout the study.

\section{Statistical analysis}

104 patients in each of the two treatment groups (atorvastatin and placebo) were required to reject the null hypothesis if the means of the distributions, with equal standard deviations of $70 \mathrm{~m}$, differed by at least $35 \mathrm{~m}$, with a type 1 error of 0.05 (twosided) and $95 \%$ power.

Analysis of efficacy end-points was performed by intention-totreat. Patients were excluded from the relevant efficacy analysis if they had a missing baseline value. Missing variables at 24-week for 6-min walk distance, WHO functional class, Borg dyspnoea score and haemodynamic parameters were replaced 
with the last available value carried forward unless clinical worsening or death occurred.

In the case of death, the value for 6-min walk distance was recorded as " $0 \mathrm{~m}^{\prime}$, while in the case of clinical worsening, the value was replaced with "worst value". The worst value for a patient was defined as his or her baseline value adjusted for the worst percentage change from baseline observed during the study. If a patient discontinued the study due to clinical worsening or death and did not have an assessment at the time of premature discontinuation, WHO functional class and Borg dyspnoea score were replaced by the worst value observed in the present study.

Patients who had no haemodynamic parameters (mean right atrial pressure, mean pulmonary artery pressure, cardiac index, pulmonary vascular resistance and mixed venous oxygen saturation) at the time of discontinuation due to clinical worsening or death were replaced using worst value defined as his or her baseline value corrected for the highest percentage of deterioration from baseline at the week 24 time point.

For low-density lipoprotein, missing values were replaced with expected variables calculated on the average percentage change between baseline and 24 weeks observed in the whole group. No imputation rule was applied to laboratory variables in patients who died during study period.

Comparison of the atorvastatin and placebo-treated groups for change in 6-min walk distance, Borg dyspnoea score, lowdensity lipoprotein level and haemodynamics parameters (mean right atrial pressure, mean pulmonary arterial pressure, cardiac index, pulmonary vascular resistance and mixed venous oxygen saturation) was made using the Wilcoxon rank sum test. The change in 6-min walk distance was analysed in subgroups defined by demographic, cause of disease and prognostic variables. Change from baseline in WHO functional class at week 24 was analysed categorically with a 3-point scale: $<0$ (improved), 0 (no change), $>0$ (worsened). The proportions of patients with no clinical worsening were calculated using the Kaplan-Meier method. Time from randomisation to the first occurrence of clinical worsening was compared with log-rank test. Subjects who completed the study or discontinued early without clinical worsening were considered censored at the time of study completion. Safety data were summarised descriptively. Analysis of PAH subgroups was retrospective. Data are expressed as mean \pm SD or mean $(95 \%$ confidence interval) or number of patients. Mean change with $95 \%$ confidence interval in haemodynamic variables were estimated by mean of differences of these variables from baseline to week 24 in treatment group minus that in placebo group. All reported p-values are two-sided. All data analyses were performed using SAS 9.1 (SAS Institute Inc.).

\section{RESULTS}

\section{Baseline patient characteristics}

A total of 220 patients were randomised to atorvastatin or placebo groups (fig. 1). Patient demographics and baseline characteristics were well matched between treatment groups, except for a higher proportion of $\mathrm{PAH}$ associated with congenital heart disease in the atorvastatin group (table 1$)$. The majority $(44 \%)$ of patients had $\mathrm{PAH}$ associated with congenital heart disease. During the 24week study period, 14 patients (11 patients in the atorvastatin group and three patients in the placebo group) reduced their dose of study medication from $10 \mathrm{mg}$ to $5 \mathrm{mg}$ daily. 16 patients (five patients in the atorvastatin group and 11 patients in the placebo group) up-titrated their dose of study medication from $10 \mathrm{mg}$ to $20 \mathrm{mg}$ daily after the initial 4 weeks of therapy.

\section{Exercise capacity}

After 24 weeks of treatment, 6-min walk distance decreased by $16.6 \mathrm{~m}$ in the atorvastatin group and $14.1 \mathrm{~m}$ in the placebo group. The mean placebo-corrected treatment effect at week 24 was $-2.5 \mathrm{~m}(95 \% \mathrm{CI}-38-33 ; \mathrm{p}=0.96)$ (fig. 2).

\section{Borg dyspnoea score}

There was no significant change in Borg index from baseline in either the atorvastatin $3(2-4)$ to $3(2-5)$ or placebo $3(2-4)$ to 3 (2-5) treated groups.

\section{Haemodynamic parameters}

Changes in haemodynamic parameters are shown in table 2. The patients treated with atorvastatin showed an increase in right atrial pressure, mean pulmonary arterial pressure, pulmonary vascular resistance from baseline, and a decrease in the cardiac index and mixed venous oxygen saturation. These changes did not differ significantly from those patients treated with placebo.

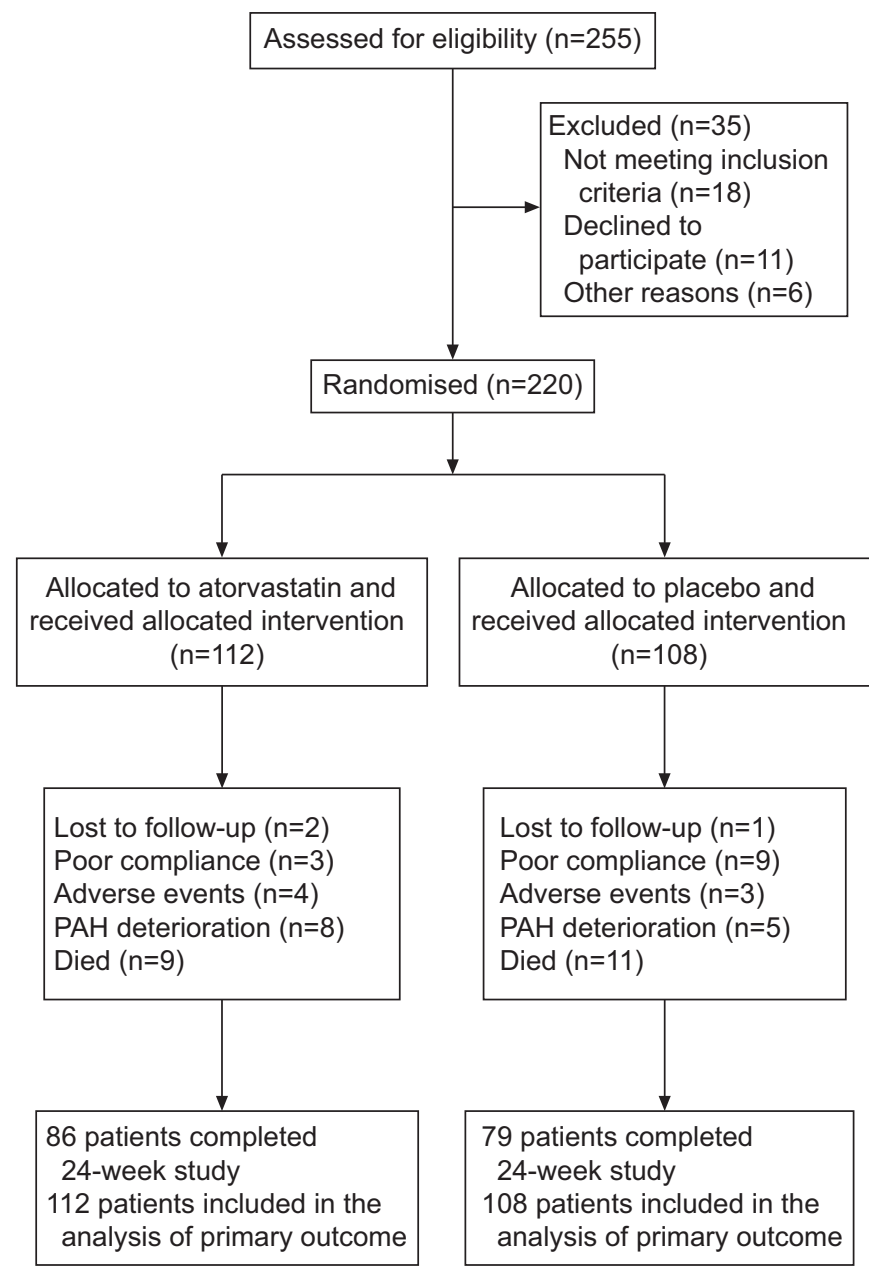

FIGURE 1. Numbers of patients enrolled in the 24-week study. PAH pulmonary arterial hypertension. 


\begin{tabular}{|c|c|c|}
\hline $\begin{array}{l}\text { Baseline clinical, function } \\
\text { haemodynamic characte }\end{array}$ & $\begin{array}{l}\text { hal, laborato } \\
\text { ristics of the }\end{array}$ & $\begin{array}{l}\text { and } \\
\text { atients }\end{array}$ \\
\hline Characteristics & Atorvastatin & Placebo \\
\hline Subjects & 112 & 108 \\
\hline Age yrs & $35 \pm 13$ & $37 \pm 13$ \\
\hline \multicolumn{3}{|l|}{ Sex } \\
\hline Female & $79(71)$ & $65(60)$ \\
\hline Male & $33(29)$ & $43(40)$ \\
\hline Weight kg & $54 \pm 12$ & $57 \pm 11$ \\
\hline Systolic blood pressure $\mathrm{mmHg}$ & $112 \pm 15$ & $113 \pm 15$ \\
\hline Diastolic blood pressure $\mathrm{mmHg}$ & $75 \pm 12$ & $73 \pm 12$ \\
\hline Heart rate beats $\cdot \mathrm{min}^{-1}$ & $80 \pm 11.8$ & $81 \pm 10.6$ \\
\hline \multicolumn{3}{|l|}{ Cause of pulmonary hypertension } \\
\hline Idiopathic & $30(27)$ & $34(32)$ \\
\hline Congenital heart disease & $59(53)$ & $38(35)$ \\
\hline Atrialseptal defect & 18 & 5 \\
\hline Ventricular septal defect & 22 & 22 \\
\hline Patent ductus arteriosus & 12 & 5 \\
\hline Other & 7 & 6 \\
\hline Connective-tissue disease & $8(7)$ & $13(12)$ \\
\hline CTEPH & $15(13)$ & $23(21)$ \\
\hline \multicolumn{3}{|l|}{ WHO functional class } \\
\hline ॥ & $70(63)$ & $71(66)$ \\
\hline III & $42(37)$ & $37(34)$ \\
\hline Six-min walk distance $\mathrm{m}$ & $355 \pm 74$ & $346 \pm 84$ \\
\hline Borg dyspnoea index & $3(2-4)$ & $3(2-4)$ \\
\hline Mixed venous oxygen saturation $\%$ & $65 \pm 8$ & $66 \pm 8$ \\
\hline Total cholesterol $\mathrm{mmol} \cdot \mathrm{L}^{-1}$ & $4.0 \pm 0.9$ & $4.2 \pm 1.0$ \\
\hline Low-density lipoprotein $\mathrm{mmol} \cdot \mathrm{L}^{-1}$ & $2.3 \pm 0.7$ & $2.3 \pm 0.8$ \\
\hline \multicolumn{3}{|l|}{ Haemodynamic parameters } \\
\hline Right atrial pressure $\mathrm{mmHg}$ & $9 \pm 6$ & $10 \pm 6$ \\
\hline Mean pulmonary arterial pressure $\mathrm{mmHg}$ & $69 \pm 19$ & $66 \pm 20$ \\
\hline Pulmonary capillary wedge pressure $\mathrm{mmHg}$ & $9 \pm 4$ & $10 \pm 4$ \\
\hline Cardiac output L. $\mathrm{min}^{-1}$ & $3.8 \pm 1.4$ & $4.1 \pm 1.7$ \\
\hline Cardiac index $L \cdot \mathrm{min}^{-1} \cdot \mathrm{m}^{-2}$ & $2.4 \pm 0.8$ & $2.6 \pm 1$ \\
\hline Pulmonary vascular resistance dyn $\cdot \mathrm{s} \cdot \mathrm{cm}^{-5}$ & $1633 \pm 745$ & $1456 \pm 699$ \\
\hline \multicolumn{3}{|l|}{ Concomitant medication } \\
\hline Digoxin & $67(60)$ & $58(54)$ \\
\hline Diuretics & $87(78)$ & $84(78)$ \\
\hline Warfarin & $64(57)$ & $60(56)$ \\
\hline
\end{tabular}

Data are presented as $\mathrm{n}$, mean $\pm \mathrm{SD}, \mathrm{n}$ (\%) or median (interquartile range). CTEPH: chronic thromboembolic pulmonary hypertension; WHO: World Health Organization

\section{WHO functional class}

In atorvastatin-treated patients, $14 \%$ improved WHO functional class and 19\% deteriorated. In placebo-treated patients, $8 \%$ improved and $18 \%$ deteriorated. There was no significant difference between the two treatment groups in the proportion of patients who improved, remained stable or deteriorated in WHO functional class ( $\mathrm{p}=0.34$ ) (fig. 3 ).

\section{Clinical worsening}

There was no significant difference between the atorvastatin and placebo groups in time to clinical worsening (fig. 4). The proportion of each component of clinical worsening (death

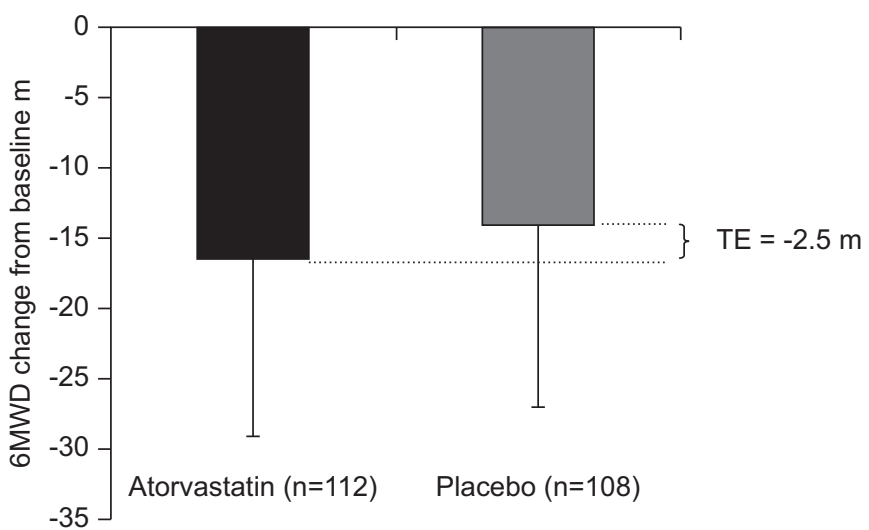

FIGURE 2. Changes from baseline of 6-min walk distance (6MWD) in atorvastatin and placebo groups. Data are presented as mean \pm SE. TE: treatment effect.

$(p=0.6)$, hospitalisations for worsening of pulmonary hypertension $(p=0.3)$ or initiation of PAH targeted therapy $(p=1.0))$ was similar between the atorvastatin group and the placebo group.

\section{Laboratory variables}

Baseline low-density lipoprotein levels were $2.3 \pm 0.7 \mathrm{mmol} \cdot \mathrm{L}^{-1}$ in the atorvastatin group and $2.3 \pm 0.8 \mathrm{mmol} \cdot \mathrm{L}^{-1}$ in the placebo group, respectively $(\mathrm{p}=0.7)$. At week 24 these were $1.8 \pm 0.6$ and $2.2 \pm 0.8 \mathrm{mmol} \cdot \mathrm{L}^{-1}$, respectively. The mean placebo-corrected treatment effects at week 24 were $-0.4 \mathrm{mmol} \cdot \mathrm{L}^{-1}(95 \% \mathrm{CI}$ $-0.6-0.2 ; \mathrm{p}<0.0001)$, indicating good compliance with atorvastatin treatment.

\section{Response in patient subgroups}

The primary efficacy measure and other variables were evaluated retrospectively in different subgroups of patients (fig. 5, tables 3 and 4). With the exception of a reduction in plasma low-density lipoprotein levels, atorvastatin was not associated with any significant effect in any subgroup examined.

\section{Safety}

The most frequent adverse events in both groups are shown in table 5. The total number of adverse events was similar in the atorvastatin group and the placebo group. 51 patients reported 79 adverse events. The common adverse events included indigestion, liver function abnormalities, irregular menstrual cycles, abdominal distention, abdominal pain, paresthesia, dizziness, myalgia and insomnia. Discontinuation rates were $23.2 \%$ and $26.9 \%$ in the two treatment groups: 26 patients receiving atorvastatin and 29 patients receiving placebo. Of the patients treated with atorvastatin, 17 discontinued because of clinical worsening (nine patients died during follow-up), nine patients discontinued for other reasons (lost follow-up $(n=2)$, poor compliance $(n=3)$, adverse events $(n=4))$. Of the patients treated with placebo, 16 discontinued because of clinical worsening (11 patients died during follow-up), 13 patients discontinued for other reasons (lost follow-up $(n=1)$, poor compliance $(n=9)$, adverse events $(n=3))$. The adverse events leading to withdrawal included abnormal liver function (two in atorvastatin group and one in placebo group), indigestion (one in atorvastatin group and one in placebo group) and dizziness (one in atorvastatin group and one in placebo group). 
TABLE 2 Mean change in haemodynamic variables from baseline to week 24

\begin{tabular}{|c|c|c|c|c|}
\hline Right atrial pressure $\mathrm{mmHg}$ & $3(2-5)$ & $3(2-5)$ & $0(-2-3)$ & 0.78 \\
\hline Cardiac index $\mathrm{L} \cdot \mathrm{min}^{-1} \cdot \mathrm{m}^{-2}$ & $-0.1(-0.2-0)$ & $-0.2(-0.3-0)$ & $0.1(-0.1-0.3)$ & 0.19 \\
\hline Pulmonary vascular resistance, dyn $\cdot \mathbf{s} \cdot \mathrm{cm}^{-5}$ & $203(42-364)$ & $287(132-442)$ & $-84(-306-138)$ & 0.15 \\
\hline Mixed venous oxygen saturation \% & $-3(-5--2)$ & $-5(-7--4)$ & $2(0-4)$ & 0.06 \\
\hline
\end{tabular}

Data are presented as mean (95\% confidence interval). ${ }^{\#}: \mathrm{n}=112 ; \bullet: \mathrm{n}=108{ }^{+}$: Wilcoxon test. The number of patients per treatment group varied slightly for each parameter because of missing assessments.

20 patients died during follow-up. Of these, 12 had idiopathic $\mathrm{PAH}$, four had PAH associated with congenital heart disease (CHD)-PAH, two had PAH associated with connective tissue disorder (CTD)-PAH and two had CTEPH. 14 (70\%) patients were in WHO functional class III. Of the nine non-survivors in the atorvastatin group, five patients died of right-sided heart failure, three patients died suddenly and one patient committed suicide. Of the 11 non-survivors in the placebo group, 10 patients died of right-sided heart failure, one patient who had PAH associated with connective tissue disease died of diffuse intravascular clotting. No death was considered to be related to the study treatment.

\section{DISCUSSION}

This is the largest study to date evaluating the effect of statin treatment in patients with pulmonary hypertension and the only study with haemodynamic data. The underlying rationale, based on animal studies, was that atorvastatin would reduce pulmonary vascular resistance and so improve exercise capacity. Atorvastatin $10 \mathrm{mg}$ daily was safe and reasonably well tolerated in this study population and significantly reduced circulating cholesterol levels but had no significant impact on 6-min walk distance, cardio-pulmonary haemodynamics or survival at 6 months.

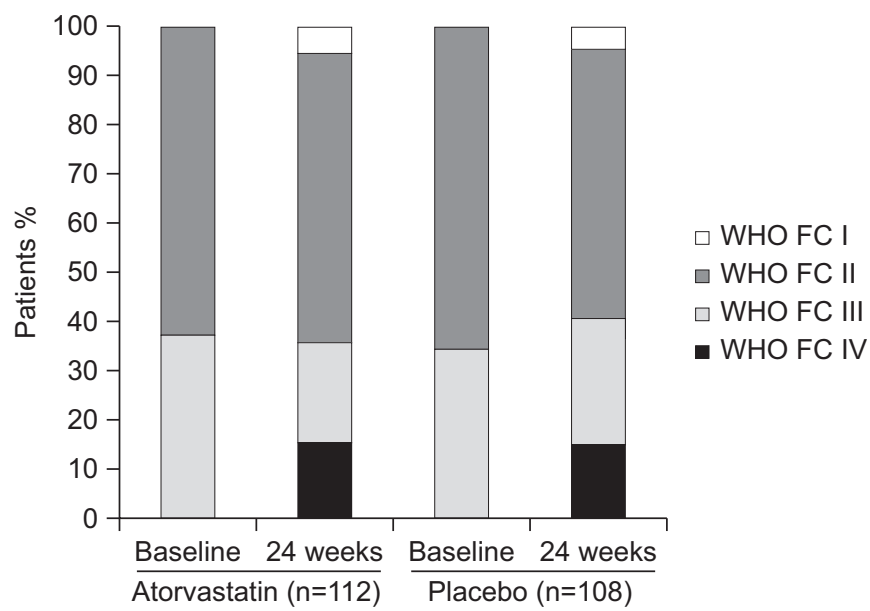

FIGURE 3. Change in World Health Organization (WHO) functional class (FC) from baseline to week 24 in the atorvastatin and placebo groups. The treatment effect of atorvastatin on WHO FC was not statistically significant.
The findings are at variance with the conclusions of a number of studies using animal models. Treatment with statins, mostly simvastatin has, with few exceptions, resulted in the prevention and reversal of pulmonary hypertension secondary to hypoxia and monocrotaline [8-14, 16]. One study showed that atorvastatin did not decrease pulmonary hypertension significantly in the monocrotaline model [13]. Our findings are consistent with two recent studies with simvastatin in patients with $\mathrm{PAH}$, which reported no effect on 6-min walk distance at 6 months [20, 21].

While statin treatment may enhance the effect of local and circulating vasorelaxant factors, for example by increasing the expression of endothelial nitric oxide synthase [9, 17], the main mechanism of action in animal models is thought to be through inhibiting cell proliferation and promoting apoptosis. Unlike changes in vasomotor tone, structural changes may take time to effect a measurable change in pulmonary haemodynamics and exercise capacity. The predominant subgroup of patients included in this study $(>40 \%)$ had co-existing congenital heart disease. The natural history in this subgroup is one of gradual decline, compared with idiopathic PAH [26]. Against a slow deterioration, a drug that acts to arrest rather than reverse pulmonary vascular modelling may need longer than 6 months to demonstrate any efficacy. But no benefit was detected in the

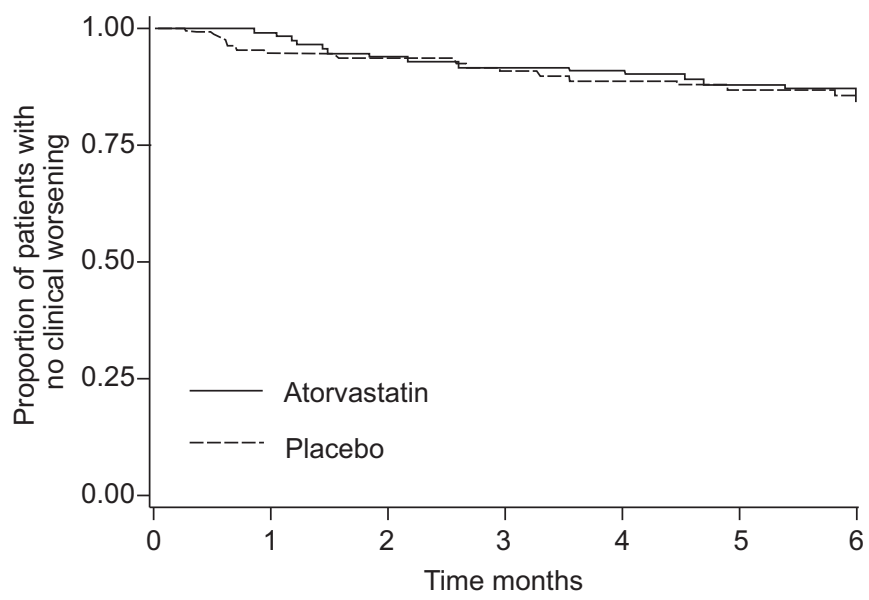

FIGURE 4. Kaplan-Meier estimates of the proportion of patients without clinical worsening. $p=0.98$ for the comparison of atorvastatin with placebo. 


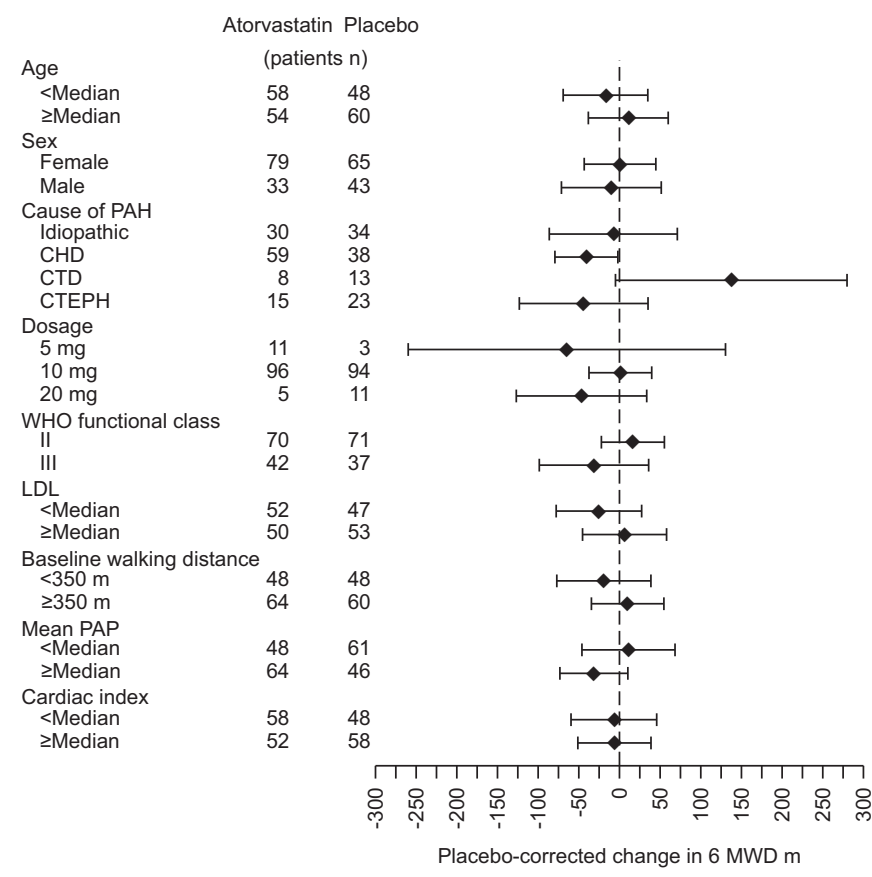

FIGURE 5. Effects of treatment on the 6-min walk distance (6MWD) from baseline to week 24 in patient subgroups. Bars represent 95\% confidence interval. PAH: pulmonary arterial hypertension; CHD: congenital heart disease; CTD: connective tissue disease; CTEPH: chronic thromboembolic pulmonary hypertension; WHO: World Health Organization; LDL: low-density lipoprotein; PAP: pulmonary artery pressure.

combined subgroup of patients with idiopathic $\mathrm{PAH}$ and $\mathrm{PAH}$ associated with connective tissue disease, a subgroup that typically exhibits a more rapid decline with time.

A strength of this study is that patients were receiving supportive medication, in the form of diuretics, digoxin and warfarin, but no PAH-targeted therapy, allowing atorvastatin to be evaluated without the confounding effects of concomitant medication. Modern targeted therapy is expensive and sildenafil is not licensed for use in pulmonary hypertension in
China. Accurate figures are difficult to obtain but it is estimated that only $20 \%$ of patients in China have access to modern therapies. Unfortunately, this study does not support the use of atorvastatin as a low-cost option for treating pulmonary hypertension.

There are several limitations to this study. One is the dose of atorvastatin used, which at $10 \mathrm{mg}$ per day is at the lower end of the licensed dose for hypercholesterolaemia. The dose of atorvastatin selected was effective at reducing plasma cholesterol levels, a marker of the effect of the drug on isoprenoid synthesis. There was concern that higher doses would produce unacceptably low cholesterol levels in this ethnic group and produce side-effects, such as elevated liver transaminase levels [27] and gastrointestinal discomfort, which would limit the translation of any benefit into clinical practice. Statins may influence pulmonary vascular biology by mechanisms other than inhibition of HMG CoA reductase [28] and a higher dose of atorvastatin may have shown greater efficacy but the present signal from atorvastatin $10 \mathrm{mg}$ is not encouraging.

Another limitation is that the study population comprised a mixture of idiopathic PAH, CHD-PAH, CTD-PAH and CTEPH. Nonetheless, while each may have a different pathobiology and natural history, histopathologic studies suggest a number of similarities in the vascular changes in CTEPH and CHD-PAH to those seen in idiopathic PAH [29, 30]. As in $\mathrm{PAH}$, recent work also suggests a role for inflammation in the genesis of CTEPH [31].

It is also recognised that the majority of patients recruited to the study were in WHO functional class II. Approved treatments, such as bosentan, which have benefits in advanced stage $\mathrm{PAH}$, have not demonstrated an improvement in 6-min walk distance in early stage disease [32].

The study also suffered from a relatively large dropout rate, equal in both arms, requiring imputation to address missing values. However, the results and conclusions were similar after per-protocol analysis (data not shown).

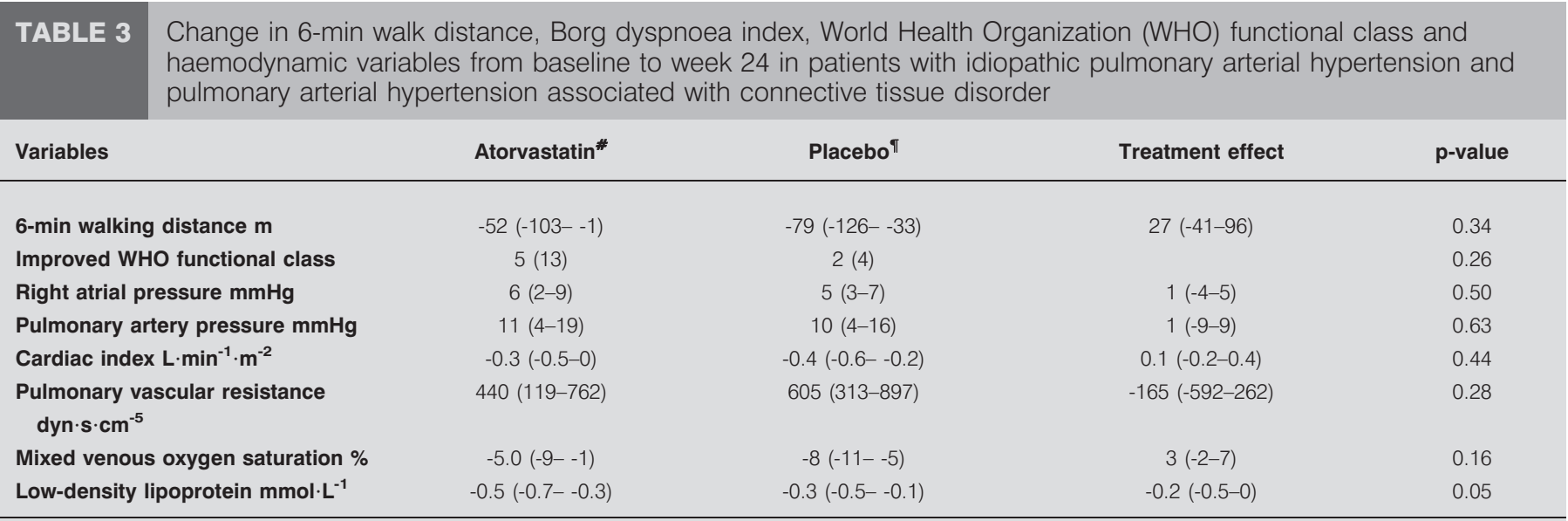

Data are presented as mean (95\% confidence interval) or $n(\%)$, unless otherwise stated. The number of patients per treatment group varied slightly for each parameter because of missing assessments. ${ }^{*}: \mathrm{n}=38 ;{ }^{\bullet}: \mathrm{n}=47$. 
TABLE 4 Change in 6-min walking distance, Borg dyspnoea index, World Health Organization (WHO) functional class and haemodynamic variables from baseline to week 24 in patients with pulmonary arterial hypertension associated with congenital heart disease

\begin{tabular}{|c|c|c|c|c|}
\hline Variables & Atorvastatin $\#$ & Placebo $\pi$ & Treatment effect & p-value \\
\hline 6-min walking distance $m$ & $10(-19-39)$ & $50(31-69)$ & $-40(-79--1)$ & 0.19 \\
\hline Right atrial pressure $\mathrm{mmHg}$ & $3(1-5)$ & $0(-2-2)$ & $3(-1-6)$ & 0.15 \\
\hline Pulmonary artery pressure $\mathrm{mmHg}$ & $0(-5-4)$ & $-5(-10-0)$ & $5(-2-12)$ & 0.12 \\
\hline Cardiac index L $\min ^{-1} \cdot \mathrm{m}^{-2}$ & $0(-0.1-0.2)$ & $0(-0.3-0.3)$ & $0(-0.3-0.4)$ & 0.28 \\
\hline Mixed venous oxygen saturation \% & $-2(-4-0)$ & $-3(-5--1)$ & $1(-2-4)$ & 0.19 \\
\hline Low-density lipoprotein $\mathrm{mmol} \cdot \mathrm{L}^{-1}$ & $-0.6(-0.8--0.4)$ & $0.05(-0.2-0.3)$ & $-0.6(-0.9--0.3)$ & $<0.001$ \\
\hline
\end{tabular}

Data are presented as mean (95\% confidence interval) or $\mathrm{n}(\%)$, unless otherwise stated. The number of patients per treatment group varied slightly for each parameter because of missing assessments. WHO: World Health Organization. ${ }^{*}: n=59 ; ": n=38$.

Rosuvastatin has been shown to reduce cardiovascular events in patients with normal cholesterol but elevated C-reactive protein (CRP) levels [33]. CRP levels have been reported to predict outcome in PAH and QUARCK et al. [34] have reported that PAH patients normalising their CRP levels on treatment (assigned as responders) showed a concomitant decrease in New York Heart Association functional class and increase in cardiac index. It might be anticipated that the effects of statins might be more pronounced in patients with higher CRP levels but this was not tested in our study.

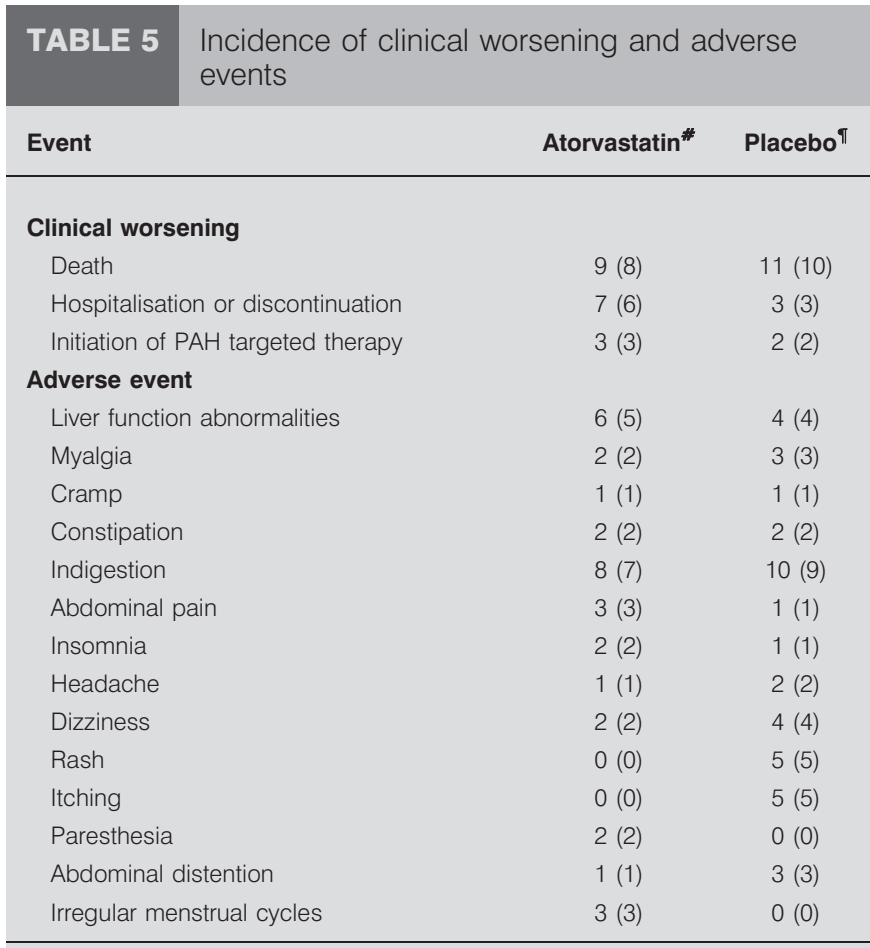

Data are presented as $n$ (\%). PAH: pulmonary arterial hypertension. ${ }^{*}: n=112$; ๑ $n=108$.
In summary, there is no evidence that 6 months of treatment with atorvastatin $10 \mathrm{mg}$ daily targets directly pulmonary vascular disease in humans. It may be used safely in this patient population when clinically indicated to reduce cholesterol levels but should not be prescribed as a specific treatment for pulmonary hypertension.

\section{SUPPORT STATEMENT}

This study was funded by a national grant from the Ministry of Science and Technology (2006BAI01A07), China, and the Capital Development Scientific Fund (2005-1018), Beijing, China.

\section{CLINICAL TRIAL}

This trial was registered at Clinical Trial.gov with identifier number NCT00615823.

\section{STATEMENT OF INTEREST}

None declared.

\section{ACKNOWLEDGEMENTS}

The authors assume full responsibility for the completeness and accuracy of the content of the manuscript. J-G. He led the project as principal investigator and takes full responsibility for the integrity of the data.

The authors would like to acknowledge the study group participants: G-Y. Wang, Beijing 301 Hospital, Beijing; H. Cao, The First Affiliated Hospital of Fujian Medical University, Fujian; D-X. Zhou, Zhongshan Hospital Fudan University, Shanghai; G-C. Zhang, Wuhan Asia Heart Hospital, Hubei; X-Y. Zhu, D-Z. Zhang, General Hospital of Shenyang Military Region, Liaoning; J-Y. Shen, Renji Hospital, Shanghai; C. Chen, S-A. Huang, The First Affiliated Hospital, Guangdong Medical College, Guangdong; W-F. Wu, K. Huang, The First Affiliated Hospital, Guangxi Medical College, Guangxi; D-J. Wang, Nanjing Drum Tower Hospital, Jiangsu; L-M. Wang, G-Q. Wu, Tongji Hospital, Shanghai; Y-H. Yang, Beijing Chao-Yang Hospital, Beijing; L-H. Wang, Sir Run Run Shaw Hospital, Zhejiang; H. Wu, Changhai Hospital, Shanghai; Z-Z. Cheng, The First Affiliated Hospital, Qingdao Medical University, Shandong; Y-Q. Ji, The First Affiliated Hospital, Dalian Medical University, Liaoning; H-Y. Tian, Y-J. Guo, The First Affiliated Hospital, Xi'an Medical University, Shanxi; B-X. Wu, The First Affiliated Hospital, Haerbin Medical University, Heilongjiang; M-T. Li, Peking Union Medical College Hospital, Beijing; Y-J. Xu, H-S. Zeng, S-X. Hu, Tongji Hospital, HuBei; G-H. Wu, C. Zhang, The First 
Affiliated Hospital, Jining Medical College, Shandong; Z-Q. Zheng, The First Affiliated Hospital of Nanchang University, Jiangxi; A-K. Lv, Shanghai Ruijin Hospital, Shanghai; H. Gu, Anzhen Hospital, Capital Medical College, Beijing; P. Jiang, Jilin Province People's Hospital, Jilin; T-Y. Sun, Beijing Hospital, Beijing; Z-W. Yang, Tianjin Medical University General Hospital, Tianjing; R. Yang, MD, Jiangsu Province People's Hospital, Jiangsu; T-L. Yang, Z-X. Yu, Q. Liu, Xiangya Hospital Central-South University, Hunan; all China.

\section{REFERENCES}

1 Archer SL, Weir EK, Wilkins MR. Basic science of pulmonary arterial hypertension for clinicians: new concepts and experimental therapies. Circulation 2010; 121: 2045-2066.

2 Benza RL, Miller DP, Gomberg-Maitland M, et al. Predicting survival in pulmonary arterial hypertension: insights from the Registry to Evaluate Early and Long-Term Pulmonary Arterial Hypertension Disease Management (REVEAL). Circulation 2010; 122: $164-172$.

3 Humbert M, Sitbon O, Chaouat A, et al. Survival in patients with idiopathic, familial, and anorexigen-associated pulmonary arterial hypertension in the modern management era. Circulation 2010; 122: 156-163.

4 Zhang R, Dai LZ, Xie WP, et al. Survival of Chinese patients with pulmonary arterial hypertension in the modern treatment era. Chest 2011; 140: 301-309.

5 McLaughlin VV, Archer SL, Badesch DB, et al. ACCF/AHA 2009 expert consensus document on pulmonary hypertension a report of the American College of Cardiology Foundation Task Force on Expert Consensus Documents and the American Heart Association developed in collaboration with the American College of Chest Physicians; American Thoracic Society, Inc.; and the Pulmonary Hypertension Association. J Am Coll Cardiol 2009; 53: 1573-1619.

6 Wang CY, Liu PY, Liao JK. Pleiotropic effects of statin therapy: molecular mechanisms and clinical results. Trends Mol Med 2008; 14: 37-44.

7 Xie L, Lin P, Xie H, et al. Effects of atorvastatin and losartan on monocrotaline-induced pulmonary artery remodeling in rats. Clin Exp Hypertens 2010; 32: 547-554.

8 Girgis RE, Li D, Zhan X, et al. Attenuation of chronic hypoxic pulmonary hypertension by simvastatin. Am J Physiol Heart Circ Physiol 2003; 285: H938-H945.

9 Guerard P, Rakotoniaina Z, Goirand F, et al. The HMG-CoA reductase inhibitor, pravastatin, prevents the development of monocrotaline-induced pulmonary hypertension in the rat through reduction of endothelial cell apoptosis and overexpression of eNOS. Naunyn Schmiedebergs Arch Pharmacol 2006; 373: 401-414.

10 Laudi S, Trump S, Schmitz V, et al. Serotonin transporter protein in pulmonary hypertensive rats treated with atorvastatin. Am J Physiol Lung Cell Mol Physiol 2007; 293: L630-L638.

11 Murata $\mathrm{T}$, Kinoshita $\mathrm{K}$, Hori M, et al. Statin protects endothelial nitric oxide synthase activity in hypoxia-induced pulmonary hypertension. Arterioscler Thromb Vasc Biol 2005; 25: 2335-2342.

12 Nishimura T, Faul JL, Berry GJ, et al. Simvastatin attenuates smooth muscle neointimal proliferation and pulmonary hypertension in rats. Am J Respir Crit Care Med 2002; 166: 1403-1408.

13 Rakotoniaina $Z$, Guerard P, Lirussi F, et al. The protective effect of HMG-CoA reductase inhibitors against monocrotaline-induced pulmonary hypertension in the rat might not be a class effect: comparison of pravastatin and atorvastatin. Naunyn Schmiedebergs Arch Pharmacol 2006; 374: 195-206.
14 Satoh K, Fukumoto Y, Nakano M, et al. Statin ameliorates hypoxiainduced pulmonary hypertension associated with down-regulated stromal cell-derived factor-1. Cardiovasc Res 2009; 81: 226-234.

15 Nishimura T, Vaszar LT, Faul JL, et al. Simvastatin rescues rats from fatal pulmonary hypertension by inducing apoptosis of neointimal smooth muscle cells. Circulation 2003; 108: 1640-1645.

16 Girgis RE, Mozammel S, Champion HC, et al. Regression of chronic hypoxic pulmonary hypertension by simvastatin. Am J Physiol Lung Cell Mol Physiol 2007; 292: L1105-L1110.

17 Zhao L, Sebkhi A, Ali O, et al. Simvastatin and sildenafil combine to attenuate pulmonary hypertension. Eur Respir J 2009; 34: 948-957.

18 Gao YF, Zhu XD, Shi DM, et al. The effects of atorvastatin on pulmonary arterial hypertension and expression of p38, p27, and Jab1 in rats. Int J Mol Med 2010; 26: 541-547.

19 Kao PN. Simvastatin treatment of pulmonary hypertension: an observational case series. Chest 2005; 127: 1446-1452.

20 Wilkins MR, Ali O, Bradlow W, et al. Simvastatin as a treatment for pulmonary hypertension trial. Am J Respir Crit Care Med 2010; 181: 1106-1113.

21 Kawut SM, Bagiella E, Lederer DJ, et al. Randomized clinical trial of aspirin and simvastatin for pulmonary arterial hypertension: ASA-STAT. Circulation 2011; 123: 2985-2993.

22 Barreto AC, Maeda NY, Soares RP, et al. Rosuvastatin and vascular dysfunction markers in pulmonary arterial hypertension: a placebo-controlled study. Braz J Med Biol Res 2008; 41: 657-663.

23 ATS statement. guidelines for the six-minute walk test. Am J Respir Crit Care Med 2002; 166: 111-117.

24 McGoon M, Gutterman D, Steen V, et al. Screening, early detection, and diagnosis of pulmonary arterial hypertension: ACCP evidence-based clinical practice guidelines. Chest 2004; 126: 14S-34S.

25 Jing ZC, Jiang X, Han ZY, et al. Iloprost for pulmonary vasodilator testing in idiopathic pulmonary arterial hypertension. Eur Respir J 2009; 33: 1354-1360.

26 McLaughlin VV, Presberg KW, Doyle RL, et al. Prognosis of pulmonary arterial hypertension: ACCP evidence-based clinical practice guidelines. Chest 2004; 126: Suppl. 1, 78S-92S.

27 LaRosa JC, Grundy SM, Waters DD, et al. Intensive lipid lowering with atorvastatin in patients with stable coronary disease. $N$ Engl J Med 2005; 352: 1425-1435.

28 Weitz-Schmidt G, Welzenbach K, Brinkmann V, et al. Statins selectively inhibit leukocyte function antigen-1 by binding to a novel regulatory integrin site. Nat Med 2001; 7: 687-692.

29 Galie N, Kim NH. Pulmonary microvascular disease in chronic thromboembolic pulmonary hypertension. Proc Am Thorac Soc 2006; 3: 571-576.

30 Beghetti M, Tissot C. Pulmonary arterial hypertension in congenital heart diseases. Semin Respir Crit Care Med 2009; 30: 421-428.

31 Kimura H, Okada O, Tanabe N, et al. Plasma monocyte chemoattractant protein-1 and pulmonary vascular resistance in chronic thromboembolic pulmonary hypertension. Am J Respir Crit Care Med 2001; 164: 319-324.

32 Galie N, Rubin L, Hoeper M, et al. Treatment of patients with mildly symptomatic pulmonary arterial hypertension with bosentan (EARLY study): a double-blind, randomised controlled trial. Lancet 2008; 371: 2093-2100.

33 Glynn RJ, Danielson E, Fonseca FA, et al. A randomized trial of rosuvastatin in the prevention of venous thromboembolism. N Engl J Med 2009; 360: 1851-1861.

34 Quarck R, Nawrot T, Meyns B, et al. C-reactive protein: a new predictor of adverse outcome in pulmonary arterial hypertension. J Am Coll Cardiol 2009; 53: 1211-1218. 